\title{
Erratum to: Can the 'neuron theory' be complemented by a universal mechanism for generic neuronal differentiation
}

\author{
Uwe Ernsberger ${ }^{1}$
}

Published online: 24 April 2015

(C) Springer-Verlag Berlin Heidelberg 2015

\section{Erratum to: Cell Tissue Res (2015) 359:343-384}

DOI 10.1007/s00441-014-2049-8

Page 348

'Upon arrival at its destination, Sox 2 expression may become upregulated again,'

\section{should read}

'Upon arrival at their destination, Sox 2 expression may become upregulated again,'.

Page 349

'In combination with Sox2, class III POU proteins (Brn1, 2, 4 and Oct6) and class V (Oct3/4) but not class II (Oct2), are strongly activated.'

\section{should read}

'In combination with Sox2, class III POU proteins (Brn1, 2, 4 and Oct6) and class V (Oct3/4) but not class II (Oct2), strongly activate.' *

Page 353

'Proneural beta helix-loop-helix transcription factors: from neural progenitor to neuron'

should read
'Proneural basic helix-loop-helix transcription factors: from neural progenitor to neuron'.

Page 364

Sox9 is expressed in undifferentiated cells but absent from endocrine or differentiated endocrine and acinar cells (Seymour et al. 2007).

should read

Sox9 is expressed in pluripotent progenitors but absent from fate-restricted endocrine precursors or differentiated endocrine and acinar cells (Seymour et al. 2007).

Page 367

CGRP-, chromogranin-, synaptophysin- or neuron-specific enolase signals are missing in lungs of newborn mutant animals (Borges et al. 1997).

should read

CGRP, chromogranin, synaptophysin or neuron-specific enolase signals are missing in lungs of newborn mutant animals (Borges et al. 1997).

Let me apologize for the mistakes.

* The error has been introduced after the proof-reading stage without knowledge of the author. 